\title{
Influence of broader geographic allograft sharing on outcomes and cost in smaller lung transplant centers
}

\author{
Nathan Haywood, MD, ${ }^{\mathrm{a}} \mathrm{J}$. Hunter Mehaffey, MD, ${ }^{\mathrm{a}}$ Sarah Kilbourne, MD, ${ }^{\mathrm{b}}$ Hannah Mannem, MD,
} Max Weder, MD, ${ }^{\mathrm{b}}$ Christine Lau, MD, ${ }^{\mathrm{c}}$ Alexander S. Krupnick, MD,${ }^{\mathrm{c}}$ and Avinash Agarwal, MD ${ }^{\mathrm{d}}$

\section{ABSTRACT}

Objective: On November 24, 2017, Organ Procurement and Transplantation Network implemented a change to lung allocation replacing donor service area with a 250 nautical mile radius around donor hospitals. We sought to evaluate the experience of a small to medium size center following implementation.

Methods: Patients (47 pre and 54 post) undergoing lung transplantation were identified from institutional database from January 2016 to October 2019. Detailed chart review and analysis of institutional cost data was performed. Univariate analysis was performed to compare eras.

Results: Similar short-term mortality and primary graft dysfunction were observed between groups. Decreased local donation ( $68 \%$ vs $6 \% ; P<.001)$, increased travel distance (145 vs 235 miles; $P=.004$ ), travel cost ( $\$ 8626$ vs $\$ 14,482 ; P<.001$ ), and total procurement cost $(\$ 60,852$ vs $\$ 69,052 ; P=.001)$ were observed postimplementation. We also document an increase in waitlist mortality postimplementation (6.9 vs 31.6 per 100 patient-years; $P<.001$ ).

Conclusions: Following implementation of the new allocation policy in a small to medium size center, several changes were in accordance with policy intention. However, concerning shifts emerged, including increased waitlist mortality and resource utilization. Continued close monitoring of transplant centers stratified by size and location are paramount to maintaining global availability of lung transplantation to all Americans regardless of geographic residence or socioeconomic status. (J Thorac Cardiovasc Surg 2022;163:339-45)

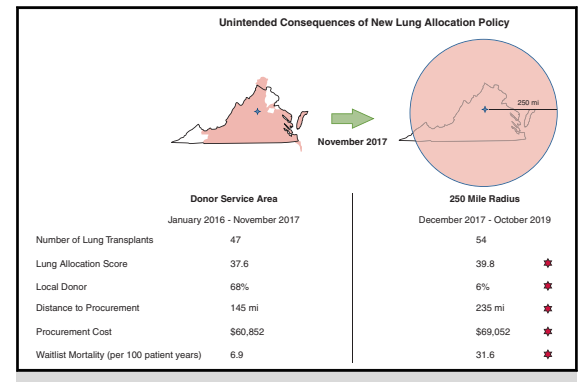

First unit of lung allocation is a 250 nautical mile radius around the donor hospital.

\section{CENTRAL MESSAGE}

Following the shift to broader

lung allograft sharing, we

demonstrate counterintuitive

changes at a smaller size center

that warrant continued moni-

toring of programs stratified by

size and location.

\begin{abstract}
PERSPECTIVE
Large center experience following the lung allocation change has been described, but information regarding smaller centers is lacking. We demonstrate counterintuitive changes in a small to medium size lung transplant center that may be representative of other similar programs. Close monitoring and adjustment is essential to maintain availability to all Americans regardless of geographic residence.
\end{abstract}

See Commentaries on pages $346,348,349$, and 350.
Lung transplantation (LT) has become an accepted and efficacious therapy for treatment of end-stage pulmonary failure. ${ }^{1}$ Due to the shortage of acceptable donors there exist

\footnotetext{
From the Divisions of ${ }^{\mathrm{a}}$ Thoracic \& Cardiovascular Surgery and ${ }^{\mathrm{d}}$ Transplantation, Department of Surgery, and ${ }^{\mathrm{b}}$ Division of Pulmonary and Critical Care Medicine, Department of Medicine, University of Virginia Health System, Charlottesville, Va; and ${ }^{\mathrm{c}}$ Division of Thoracic Surgery, Department of Surgery, University of Maryland Medical Center, Baltimore, Md.

Supported in part by the National Heart, Lung, and Blood Institute of the National Institutes of Health (grant No. T32HL007849). The content is solely the responsibility of the authors and does not represent the official views of the National Institutes of Health.
}

significant challenges associated with allocation. ${ }^{2,3}$ Historically, the first geographic unit of LT allocation was the donor service area (DSA), which divides the country into 58

Received for publication April 6, 2020; revisions received Aug 21, 2020; accepted for publication Sept 1, 2020; available ahead of print Sept 6, 2020

Address for reprints: Avinash Agarwal, MD, Division of Transplantation, Department of Surgery, University of Virginia Transplant Center, Fourth Floor, 1300 Jefferson Park Ave, Charlottesville, VA 22903 (E-mail: AA4VB@virginia.edu). $0022-5223 / \$ 36.00$

Copyright (c) 2020 by The American Association for Thoracic Surgery https://doi.org/10.1016/j.jtcvs.2020.09.008 


\section{Abbreviations and Acronyms \\ DSA $=$ donor service area \\ LAS = lung allocation score \\ LT = lung transplantation \\ $\mathrm{OPO}=$ Organ Procurement Organization \\ OPTN $=$ Organ Procurement and Transplantation Network \\ UVA $=$ University of Virginia}

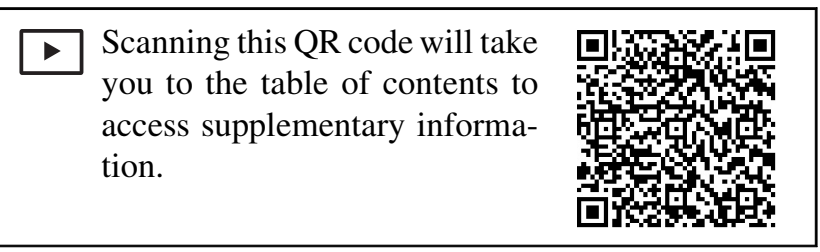

regions with defined geographic borders. ${ }^{3-6}$ However, on November 24, 2017, in response to a lawsuit from a transplant candidate in New York, an emergency action change was implemented by the Organ Procurement and Transplantation Network (OPTN) that replaced the DSA with a 250 nautical mile radius around the donor hospital. ${ }^{5,7-9}$ The OPTN recently published a 1-year report detailing national findings since the new allocation policy was implemented. ${ }^{10}$ Some of the highlights include higher lung allocation score (LAS) at transplant, longer distance between donor hospital and transplant center, and increased graft ischemia times. ${ }^{10}$

The large center experience in the post policy implementation era has recently been described..$^{9}$ Although the experience of high volume centers are critically important to understand, small to medium size transplant centers also make up a significant proportion of total LT performed. ${ }^{11,12}$ An analysis of United Network for Organ Sharing registry data from 2005 to 2010 demonstrated that $53 \%$ of LT were performed at high volume centers $(>170$ LT from 2005 to 2010 ) and $47 \%$ were performed at low to medium volume centers $(<170 \mathrm{LT}$ from 2005 to 2010$) .{ }^{11}$ Thus, close to half of Americans who receive LT are taken care of in a lower volume program. The experience of such centers, following implementation of the 250 nautical mile system has yet to be described.

The University of Virginia (UVA) is a well-established small to medium size LT center located in central Virginia. The program is the primary LT center serving central and southwestern Virginia, the latter of which is a region shown to be socioeconomically disadvantaged with lower than average access to large health care facilities. ${ }^{13}$ Eight other LT centers are located within a 250-mi radius of UVA, including Temple University, Duke University, University of Pennsylvania, University of Pittsburgh, Johns Hopkins
University, University of Maryland, University of North Carolina, and Inova Fairfax. UVA averages approximately 25 to 30 transplants per year and has performed more than 580 LTs at the time of this research. The purpose of this study was to evaluate the experience of a small to medium size LT center following implementation of the new lung allocation policy. We hypothesized increased recipient LAS, graft ischemia time, and resource utilization would occur in the postimplementation era.

\section{MATERIALS AND METHODS \\ Patient Data}

All records for patients undergoing LT from January 2016 to October 2019 were extracted from an institutional database. Patients were stratified by date of transplant: before or after lung allocation policy change (November 2, 2017) (Figure 1). Detailed chart review was performed to identify preoperative, operative, and postoperative recipient characteristics. Donor characteristics were obtained from DonorNet utilizing United Network for Organ Sharing ID and match ID to search. Primary graft dysfunction at 72 hours was assessed by examination of $\mathrm{PaO}_{2} / \mathrm{FiO}_{2}$ ratio and chest radiograph at this time. A board-certified transplant pulmonologist reviewed imaging and laboratory data. Standard International Society for Heart and Lung Transplantation grading system was used. ${ }^{14}$ Procurement location was identified via retrospective chart review. Straight-line distance, in miles, was calculated using Google Maps (Google Inc, Mountain View, Calif). Cost data, representing cost incurred, was obtained from institutional finance department. Costs were adjusted to 2019 equivalent dollars using the Centers for Medicare and Medicaid Services Inpatient Prospective Payment System adjustment for medical related inflation. During the study period there were no changes in internal policy regarding listing or organ acceptance criteria. The UVA Institutional Review Board approved this study with a waiver of patient consent due to its retrospective nature (UVA Institutional Review Board protocol No. 20528).

\section{Statistical Analysis}

Continuous variables are presented as median (interquartile range $[\mathrm{IQR}])$ and categorical variables are presented as $\mathrm{n}(\%)$. Patients were stratified by date of transplant: pre- or postimplementation of the new allocation policy. Patients were compared by univariate analysis using $\chi^{2}$ test for categorical variables and Mann-Whitney $U$ test for continuous variables. All statistical analyses were carried out using SPSS (IBM-SPSS Inc, Armonk, NY).

\section{RESULTS}

From January 2016 to October 2019, a total of 101 consecutive patients underwent LT: 47 patients before the implementation of the new allocation policy (November 24, 2017) and 54 patients after (Figure 1).

\section{Preoperative and Operative Characteristics}

Preoperative characteristics were compared between patients transplanted before and after allocation policy implementation (Table 1). There were no significant differences in the distribution of baseline demographic characteristics between groups. The 2 groups were similar in terms of home oxygen requirement, time on wait list, smoking history, cytomegalovirus positivity, and sensitization reported by panel reactive antibody. There was an increase in LAS 


\section{Unintended Consequences of New Lung Allocation Policy}

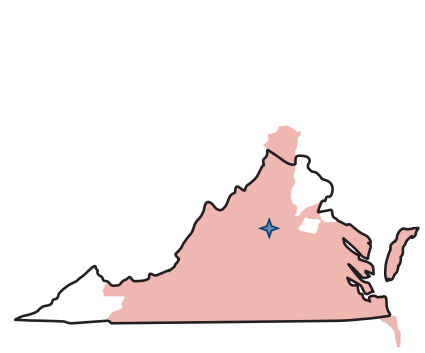

Donor Service Area

January 2016 - November 2017

$\begin{array}{ll}\text { Number of Lung Transplants } & 47 \\ \text { Lung Allocation Score } & 37.6 \\ \text { Local Donor } & 68 \% \\ \text { Distance to Procurement } & 145 \mathrm{mi} \\ \text { Procurement Cost } & \$ 60,852 \\ \text { Waitlist Mortality (per 100 patient years) } & 6.9\end{array}$
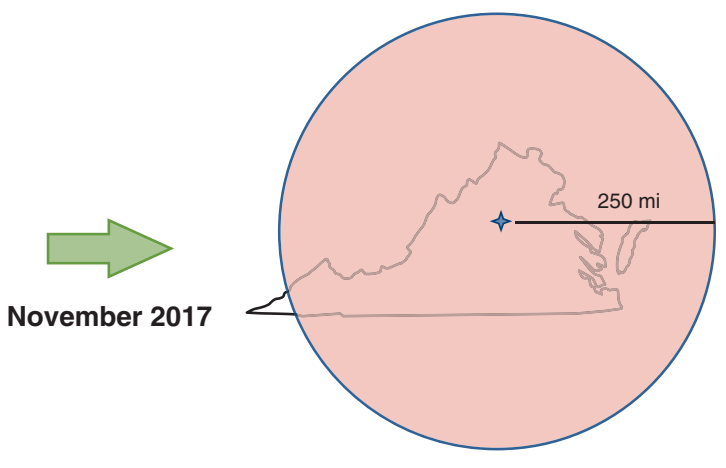

250 Mile Radius

December 2017 - October 2019

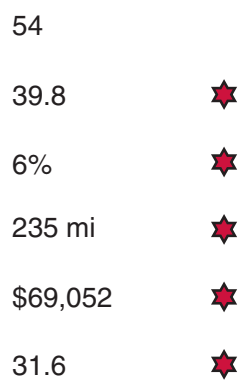

FIGURE 1. Outcomes following the shift in lung allocation from donor service area to a 250 nautical mile radius around the donor hospital. Following implementation of the new policy, findings at University of Virginia include higher lung allocation score, longer distance to procurement, decreased utilization of local donors, increased procurement cost, and increase in waitlist mortality.

postimplementation (37.6 vs $39.8 ; P=.03$ ). Similarly, donor preoperative characteristics in the 2 eras were compared (Table 1). A larger proportion of male donors were identified during the postimplementation era $(49 \%$ vs $74 \% ; P=.01$ ). Donors were similar in terms of age, best arterial oxygen tension, and cytomegalovirus positivity.

Operative characteristics pre- and postimplementation were compared (Table 1). Experiences in both eras were similar in terms of rate of bilateral versus single LT and use of ex vivo lung perfusion. Although left warm ischemia time decreased between eras (52 vs $45 \mathrm{~min}, P=.03$ ), cold ischemia time and total ischemia times were unchanged.

\section{Waitlist Characteristics}

Waitlist characteristics were compared before and after the policy change. Waitlist mortality rate increased from 6.9 to 31.6 per 100 patient-years $(P<.001)$ following implementation. In the pre era there were a total of 59 patients listed compared with 77 during the post era. The daily waitlist size increased between eras (7 patients; IQR, 6-9 patients vs 9 patients; IQR, $7-11$ patients; $P<.001$ ). In the pre era 5 patients were delisted due to either patient condition $(n=1)$ or patient preference $(n=4)$. There was 1 waitlist mortality during this time period. The patient died while an inpatient and the cause of death was acute on chronic hypoxic respiratory failure in a setting of interstitial lung disease (LAS, 76.6). In the post era there were 8 patients delisted due to either patient condition $(\mathrm{n}=6)$ or patient preference $(n=2)$. There were 5 waitlist mortalities during this era: 3 while listed and 2 shortly after delisting $(<1$ week). All mortalities occurred during inpatient stay and LAS scores were all $>80$. Cause of death in 4 patients was acute chronic respiratory failure in a setting of interstitial lung disease. In 1 patient, cause of death was acute respiratory failure in a setting of cystic fibrosis with infectious complication. Among all waitlist mortalities, only 1 patient during the post era was sensitized (panel reactive antibody, $25 \%$ ).

\section{Transplant Indication and Donor Cause of Death}

The primary indication for LT was compared pre and post policy change (Table 2). There was a higher rate of alpha 1 antitrypsin disease before implementation $(13 \%$ vs $2 \%$; $P=.03)$ and a nonsignificant increase in interstitial lung disease after implementation ( $47 \%$ vs $65 \% ; P=.07)$. Rate of chronic obstructive pulmonary disease/emphysema, cystic fibrosis, and pulmonary hypertension were similar 
TABLE 1. Preoperative and operative characteristics between eras

\begin{tabular}{|c|c|c|c|}
\hline Variable & $\begin{array}{c}\text { Prepolicy } \\
(n=47)\end{array}$ & $\begin{array}{l}\text { Postpolicy } \\
(n=54)\end{array}$ & $P$ value \\
\hline \multicolumn{4}{|l|}{$\begin{array}{l}\text { Recipient preoperative } \\
\text { characteristics }\end{array}$} \\
\hline Age & $58(50-64)$ & $59(53-65)$ & .39 \\
\hline Male & $22(47)$ & $32(59)$ & .21 \\
\hline Vent requirement & $1(2)$ & $3(6)$ & .38 \\
\hline Home oxygen* (L) & $3(2-5)$ & $4(2-6)$ & .31 \\
\hline Lung allocation score & $37.6(33.6-43.0)$ & $39.8(35.2-46.3)$ & .03 \\
\hline Time on wait list (d) & $42(17-95)$ & $38(19-81)$ & .59 \\
\hline $\begin{array}{l}\text { Smoking history } \\
\text { (pack-years) }\end{array}$ & $16(0-40)$ & $14(0-40)$ & .72 \\
\hline CMV positive & $28(60)$ & $30(56)$ & .68 \\
\hline \multicolumn{4}{|l|}{ Sensitization } \\
\hline Class I PRA > $>5 \%$ & $10(14)$ & $14(26)$ & .58 \\
\hline Class II PRA > $10 \%$ & $9(19)$ & $7(13)$ & .40 \\
\hline $\begin{array}{l}\text { Highly sensitized } \\
\qquad(\text { PRA }>80 \%)\end{array}$ & $2(4.3)$ & $0(0)$ & .13 \\
\hline \multicolumn{4}{|l|}{$\begin{array}{c}\text { Donor preoperative } \\
\text { characteristics }\end{array}$} \\
\hline Age & $34(22-53)$ & $35(25-41)$ & .88 \\
\hline Male & $23(49)$ & $40(74)$ & .01 \\
\hline $\begin{array}{l}\text { Best arterial oxygen } \\
\text { tension }\end{array}$ & $521(448-570)$ & $495(472-551)$ & .93 \\
\hline CMV positive & $33(70)$ & $34(63)$ & .44 \\
\hline DCD & $3(6)$ & $0(0)$ & .06 \\
\hline \multicolumn{4}{|l|}{ Operative characteristics } \\
\hline Bilateral transplant & $44(94)$ & $48(89)$ & .41 \\
\hline $\begin{array}{l}\mathrm{R} \text { Total ischemic time } \\
(\mathrm{min})\end{array}$ & $295(207-352)$ & $300(266-363)$ & .18 \\
\hline $\mathrm{R}$ Warm ischemic time & $51(43-62)$ & $50(42-60)$ & .76 \\
\hline $\mathrm{R}$ Cold ischemic time & $239(153-302)$ & 244 (214-309) & .21 \\
\hline L Total ischemic time & $284(233-325)$ & $257(208-339)$ & .62 \\
\hline L Warm ischemic time & $52(40-63)$ & $45(40-49)$ & .03 \\
\hline L Cold ischemic time & $231(164-267)$ & $226(163-295)$ & .79 \\
\hline EVLP & $2(4)$ & $2(4)$ & .89 \\
\hline
\end{tabular}

Values are presented as $\mathrm{n}(\%)$ or median (range). $C M V$, Cytomegalovirus; $P R A$, panel reactive antibody; $D C D$, donation after circulatory death; $R$, right; $L$, left; $E V L P$, ex vivo lung perfusion. *At rest.

between eras. Donor cause of death in the 2 groups was compared. Similar rates of drug intoxication $(17 \%$ vs $15 \% ; P=.76)$, intracranial hemorrhage $(51 \%$ vs $50 \%$;

TABLE 2. Primary indication for lung transplantation between eras

\begin{tabular}{lccc}
\hline $\begin{array}{c}\text { Primary transplant } \\
\text { indication }\end{array}$ & $\begin{array}{c}\text { Prepolicy } \\
(\mathbf{n}=\mathbf{4 7})\end{array}$ & $\begin{array}{c}\text { Postpolicy } \\
(\mathbf{n}=\mathbf{5 4})\end{array}$ & $\boldsymbol{P}$ value \\
\hline COPD/emphysema & $12(26)$ & $8(15)$ & .18 \\
Alpha 1 antitrypsin & $6(13)$ & $1(2)$ & .03 \\
Cystic fibrosis & $5(11)$ & $7(13)$ & .72 \\
Interstitial lung disease & $22(47)$ & $35(65)$ & .07 \\
Pulmonary hypertension & $1(2.1)$ & $0(0)$ & .28 \\
Other & $1(2)$ & $3(6)$ & .38 \\
\hline
\end{tabular}

Values are presented as $\mathrm{n}(\%) . C O P D$, Chronic obstructive pulmonary disease.
$P=.92)$, cardiovascular insult ( $6 \%$ vs $9 \% ; P=.59)$, gunshot wound $(17 \%$ vs $17 \% ; P=.96)$, and asphyxiation $(6 \%$ vs $6 \% ; P=.86$ ) were observed. Number of donor offers were compared before and after the policy change. The number of offers per month increased significantly between eras (24 offers; IQR, 17-33 offers vs 89 offers; IQR, 66-138 offers; $P<.001)$.

\section{Postoperative Outcomes and Resource Utilization}

Postoperative outcomes before and after the allocation policy change were compared (Table 3 ). Rate of primary graft dysfunction and short-term mortality (30- and 90day) were similar between groups. Compared with the postimplementation era, there was a significantly higher rate of local donors $(68 \%$ vs $6 \% ; P<.001)$ (Table 3$)$ during the preimplementation era. Longer travel distance (145 vs 235 miles; $P=.004)$, increased travel cost ( $\$ 7948$ vs $\$ 14,365 ; P<.001)$, and total procurement cost $(\$ 56,548$ vs $\$ 68,157 ; P<.001)$ were observed during the post era. Following inflation adjustment, there remained higher travel cost ( $\$ 8626$ vs $\$ 14,482 ; P<.001)$ and total procurement cost $(\$ 60,852$ vs 69,$052 ; P=.001)$ following the policy change. Rate of negative fly out (flying to donor hospital and declining the organ) was assessed. Similar negative fly out rate was found between eras, with $10(21 \%)$ during the pre policy era and $17(25 \%)$ postimplementation $(P=.60)$. Although length of intensive care unit stay was similar between groups, length of hospital stay was longer during the postimplementation era (18 vs 26 days; $P=.01$ ).

\section{DISCUSSION}

We present an analysis of the postimplementation experience of the new lung allocation policy in a small to medium size LT center (Figure 1 and Video 1). Although the rate of local donation decreased and travel distances increased, there was no change in total graft ischemia time, primary graft dysfunction, or short-term mortality. We found an increase in waitlist mortality rate at our institution following the policy change. Additionally, resource utilization, namely travel and procurement cost, significantly increased during the postimplementation era.

The intention of the 250 nautical mile allocation change was to replace arbitrary geographic constraints of DSAs with a broader, more equitable sharing policy. ${ }^{8}$ However, certain inequities remain, the most easily identifiable of which are geographic in nature. For example, transplant centers in coastal cities like Miami, Fla, have a geographic disadvantage in that a large percentage of the surrounding 250-mile radius is water, which certainly limits donor opportunities. Additionally, population and hospital density play an important role. Take, for example, Minneapolis, Minn, compared with Indianapolis, Ind. There are far fewer major metropolitan areas within a 250 nautical mile radius around Minneapolis compared with that around Indianapolis, which includes Chicago, Ill; St. Louis, 
TABLE 3. Postoperative outcomes and resource utilization by era

\begin{tabular}{lccc}
\hline \multicolumn{1}{c}{ Variable } & Prepolicy $(\mathbf{n}=\mathbf{4 7})$ & Postpolicy $(\mathbf{n}=\mathbf{5 4})$ & $\boldsymbol{P}$ value \\
\hline Postoperative outcome & & & \\
Primary graft dysfunction & $12(26)$ & $16(30)$ & $9(17)$ \\
Grade 3 primary graft dysfunction & $6(13)$ & $0(0)$ & .65 \\
30-d mortality & $1(2)$ & $0(0)$ & .28 \\
90-d mortality & $3(6)$ & $3(6)$ & .09 \\
Resource utilization & $32(68)$ & $235(155-285)$ & $<.001$ \\
Local donor & $145(65-393)$ & $\$ 46,035(\$ 40,800-\$ 51,050)$ \\
Distance to procurement (mi) & $\$ 48,600(\$ 48,600-\$ 48,600)$ & $\$ 14,365(\$ 9528-\$ 17,117)$ & .004 \\
Organ cost & $\$ 7948(\$ 4764-\$ 12,601)$ & $\$ 68,157(\$ 63,594-\$ 74,905)$ & \\
Travel cost & $\$ 56,548(\$ 53,364-\$ 67,645)$ & $26(18-37)$ & $10(6-22)$ \\
Total procurement cost & $18(12-31)$ & $<.001$ \\
Length of stay (d) & $7(4-20)$ & .001 \\
Length of ICU stay (d) & & .01 \\
\hline
\end{tabular}

Values are presented as $\mathrm{n}(\%)$ or median (range). ICU, Intensive care unit.

Mo; Louisville, Ky; Cincinnati, Ohio; and Columbus, Ohio. It is likely that opportunity in these 2 areas are quite different. The discussion becomes more complex when considering transplant center density as increased competition between centers limit donor opportunities per center. Thus, an ideal scenario for a high-volume center would likely be in an area with a high population density to transplant center density ratio.

Granular data are critical to understand experience variation in a postallocation era. In a small to medium size center located in central Virginia, we found that despite increased travel distance, total ischemia times were unchanged following implementation of the new allocation policy. This contradicts previous reports that demonstrate increased ischemia time along with increased travel distance. $^{9,10}$ We believe that this highlights an important distinction at UVA that may be representative of other small to moderate size LT programs. In the prepolicy era, median distance to donor hospital from our facility was 145 miles, necessitating air travel for almost all procurements, including the majority of those within our DSA not performed at UVA. For the rare occasion where auto transportation was feasible, travel distances were never $<30$ miles. We believe that despite the increase in distance traveled,

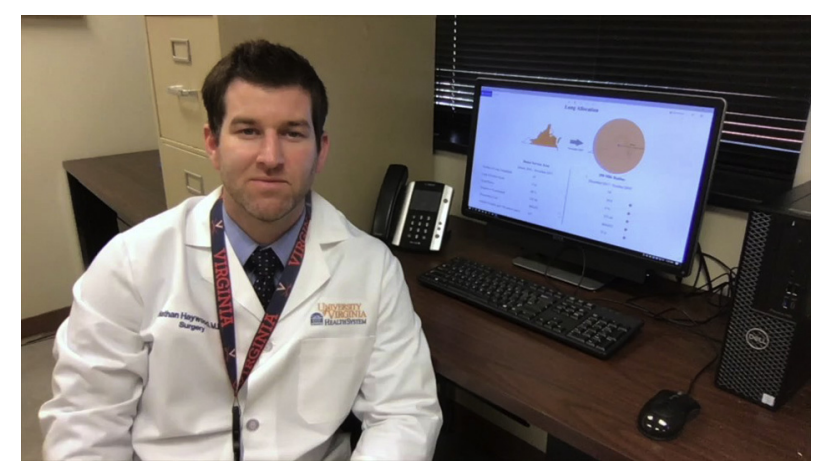

VIDEO 1. Nathan Haywood explains the importance of his work. Video available at: https://www.jtcvs.org/article/S0022-5223(20)32571-X/fulltext. the travel burden (eg, transportation to and from airport as well as loading and unloading) was largely unchanged resulting in similar graft ischemia times. In essence, and perhaps unlike the case for larger centers in densely populated areas, an increase of 50 to 100 miles of flight time did not translate to a difference in ischemic times. Many large LT centers, especially those where procurements often occur in the same city or those that utilize closely located specialized donor care facilities did not have a similar travel experience during the prepolicy era. Such data may explain the previously reported increase in ischemic times postallocation change. As we demonstrate, this may not be reflective of all programs.

We found that at our institution there was an increase in recipient LAS between eras. The OPTN 1-year report also showed a significant increase in LAS from 42.4 to $43.6,{ }^{10}$ which is similar to the magnitude of change observed in our study. Although statistically significant, the clinical significance of a 1- to 2-point increase in LAS is questionable. We are hopeful that a durable, meaningful difference will be revealed in time as the number of patients transplanted during the postimplementation era grows. Although the number of transplants at our center increased during the postallocation era, waitlist size also increased. Therefore, when normalized to median daily waitlist size, there was actually fewer LTs performed during the post era. We found that organ offers increased following implementation, which is consistent with the increase in waitlist size. Our analysis also demonstrated an increase in waitlist mortality between eras, which contradicts what has been previously reported. ${ }^{10,15}$ This may be due to difference in pathology between eras with generally less alpha 1 antitrypsin and more interstitial lung disease in post compared with pre era. Additionally, minor changes within a relatively small waitlist can have a big influence, so the apparent increase in mortality may be a reflection of the size of our waitlist. Regardless, whereas sample size is low and geographic region limits generalizability, 
this may reveal a counterintuitive trend of increased mortality in small to medium centers in certain areas because organs are redistributed to larger centers in urban areas that have an opportunity to populate their lists with sicker patients. Continued close analysis of LT programs, stratified by size and geographic location, is essential to identify such consequences during the postimplementation era.

Similar to previous reports we found no difference in short-term mortality or rate of primary graft dysfunction between groups. ${ }^{9}$ Reported rates of primary graft dysfunction are within previously published acceptable ranges. ${ }^{14}$ The rate of local donation in our study decreased by $90 \%$ postimplementation, which is a much larger drop than previously reported. ${ }^{9,10}$ For example, the OPTN 1-year report showed a $56 \%$ decrease. ${ }^{10}$ Given the decrease in local donation, distance to procurement was understandably longer (145 vs $234 \mathrm{mi}$ ) postimplementation. In the postpolicy era, the majority of procurements occurred within a 250 nautical mile radius, which is the first unit of allocation. The 1-year report from OPTN showed similar findings. ${ }^{10}$

Congruent with previous reports, we found that travel cost as well as total procurement cost was significantly higher in the postimplementation era. ${ }^{7,9,10}$ The cost of local organ procurement is made up of travel cost for the procurement team and organ acquisition cost set by the local organ procurement organization (OPO). For nonlocal procurement where a different OPO covers the donor hospital, there is an additional administrative charge by the home (recipient hospital) OPO for coordinating. ${ }^{9}$ Following inflation adjustment we found total procurement cost increased by $\$ 8200$. This increase stems from moderately increased travel expenses-inflation adjusted travel cost increased by $\$ 5856$ - as well as increased administrative overhead charges from the home OPO for coordinating nonlocal donation (\$9500). Cost reported represents direct cost and does not include indirect cost, which at academic medical centers can add an additional $40 \%$ to $50 \%$ increase. Thus, the true influence is likely even larger in magnitude. Our analysis showed a significant increase in length of stay following implementation of the new allocation system. This change is due to a more conservative discharge approach throughout the late period in response to a high 30 -day readmission rate during the early period $(25 \%)$ and is probably unrelated to the donor geographic change. The late period showed slight improvement with 30-day readmission rate of $22 \%$, and we anticipate further improvements as our approach continues to be optimized. Previous reports have raised concern over the possibility of increased negative fly outs (flying to donor hospital and declining the organ) and the influence this could have on resource utilization following implementation of the new policy. ${ }^{9}$ Our analysis showed no difference in the rate of negative fly outs. However, as a nonreimbursed activity, ${ }^{9}$ this is a concerning trend that deserves continued close attention as we move further into the postimplementation era.

Within a 250-mile radius from UVA are several population dense areas, including Washington, DC; Philadelphia, $\mathrm{Pa}$; Pittsburgh, Pa; Charlotte, NC; and Baltimore, Md. However, there are also 8 LT centers competing within this area not including centers between 250 and 500 miles away that would have an overlapping radius. This would include centers in New York and Boston. Transplant center density around UVA is primarily skewed in the northern direction. Donor location following policy change at UVA was skewed in the southern direction. For example, of 54 donors only 3 were located in Pennsylvania and 2 were located in Maryland. This may be evidence of increased competition in areas of higher center density. Many small to medium size transplant centers serve a critical need for patients from rural communities who may not have the means or resources to travel to a large center. For example, UVA, located in central Virginia is easily accessible and may be the only source of transplants for patients in southwest Virginia-a region previously shown to be medically underserved and economically disadvantaged. ${ }^{13}$ With further travel distances costs are increasing and managed care companies are not increasing reimbursement at a similar rate. The strain of this imbalance may force similar small to medium size programs to limit access or even shut down due to financial constraints that may be easily absorbed by larger, high volume centers. This could result in only a handful of large centers in America performing LT, which would be similar to the current Canadian system composed of 4 centers. ${ }^{16}$ In this theoretical scenario, American patients in rural areas would need to relocate while waitlisted—posing a significant barrier to care especially in those with limited means. Although publically funded health care partially supports waitlist relocation in Canada, ${ }^{17}$ this would likely be unsustainable in the current American health care climate. One potential opportunity to limit costs would be an increase in sharing procurement responsibilities between centers. Doing so would limit travel related cost. Travel restrictions enacted during the coronavirus disease 2019 national pandemic have, to some degree, already forced a shift toward this model. Relying on local centers to aid in procurement will require a level of trust that will take some time to develop. As such, it may be an ideal opportunity to implement remote telemonitoring technology where recipient surgeons could evaluate the organ in real time over video and help make decisions with the local procuring team. Small to medium size transplant centers are important resources that perform almost half of all LTs in the United States. ${ }^{11}$ As such, they necessitate close monitoring of postimplementation financial stressors.

This study has limitations to consider when interpreting the data. Our analysis is limited by its retrospective nature and relatively small sample size. UVA is a small to medium 
size LT center with practice patterns that may not be reflective of other centers. Although number of LT offers where evaluated, we did not assess which organs were initially offered to other centers before acceptance. Further study, stratified by region and center size, is needed to characterize number of offers before acceptance with the new allocation policy. Although referral patterns play an important role in listing, we did not directly evaluate this. Finally, the geographic setting, surrounding population density, and surrounding LT center density at UVA are unique, which limit the generalizability of findings.

\section{CONCLUSIONS}

The new lung allocation policy is congruent with the Final Rule mandate to pursue broader geographic sharing and limit arbitrary geographic borders. ${ }^{2,3}$ In this new era, the experience of LT centers, especially those of differing size and geographic location, are not homogenous. We demonstrate that unlike previous findings, graft ischemia times at our institution were unchanged despite increased travel distances postimplementation. However, similar to what has been described, our analysis found several concerning trends, most notably increases in resource utilization and an increase in wait list death rate. Such data bring up the possibility that the 2017 allocation change, designed by a court order to decrease wait list mortality, may result in unintended and counter intuitive rise in mortality in those waiting for lungs in smaller centers. Continued close monitoring and discussions regarding reimbursement are paramount, especially for small to medium size centers, moving forward.

\section{Conflict of Interest Statement}

Dr Krupnick is the founder, chief scientific officer, and a board member of Courier Therapeutics. No sponsorship or influence on this work was carried out by this company. All other authors reported no conflicts of interest.

The Journal policy requires editors and reviewers to disclose conflicts of interest and to decline handling or reviewing manuscripts for which they may have a conflict of interest. The editors and reviewers of this article have no conflicts of interest.

\section{References}

1. Valapour M, Lehr CJ, Skeans MA, Smith JM, Carrico R, Uccellini K, et al. OPTN/SRTR 2016 annual data report: lung. Am J Transplant. 2018;18(Suppl 1): 363-433.

2. Egan TM, Edwards LB. Effect of the lung allocation score on lung transplantation in the United States. J Heart Lung Transplant. 2016;35: 433-9.

3. Egan TM. How should lungs be allocated for transplant? Semin Respir Crit Care Med. 2018;39:126-37.

4. Colvin-Adams M, Valapour M, Hertz M, Heubner B, Paulson K, Dhungel V, et al. Lung and heart allocation in the United States. Am J Transplant. 2012;12: 3213-34.

5. Kosztowski M, Zhou S, Bush E, Higgins RS, Segev DL, Gentry SE. Geographic disparities in lung transplant rates. Am J Transplant. 2019;19:1491-7.

6. Snyder JJ, Salkowski N, Wey A, Pyke J, Israni AK, Kasiske BL. Organ distribution without geographic boundaries: a possible framework for organ allocation. Am J Transplant. 2018;18:2635-40.

7. OPTN Thoracic Transplantation Committee. Monitoring of the lung allocation change, 6-month report removal of DSA as a unit of allocation; 2018. Available at: https://optn.transplant.hrsa.gov/media/2517/20180621_thoracic_committee_ report_lung.pdf. Accessed September 14, 2020.

8. OPTN Thoracic Transplantation Committee. HRSA letter to OPTN; 2017, https://optn.transplant.hrsa.gov/media/2397/hrsa_letter_to_optn_20171121.pdf. Accessed September 14, 2020.

9. Puri V, Hachem RR, Frye CC, Harrison MS, Semenkovich TR, Lynch JP, et al. Unintended consequences of changes to lung allocation policy. Am J Transplant. 2019;19:2164-7.

10. OPTN Thoracic Transplantation Committee. Monitoring of the lung allocation change, 1 year report; 2019. Available at: https://optn.transplant.hrsa.gov/media/ 2815/20190116_thoracic_committee_report_lung.pdf. Accessed September 14, 2020.

11. Hayes D Jr, Tobias JD, Tumin D. Center volume and extracorporeal membrane oxygenation support at lung transplantation in the lung allocation score era. Am J Respir Crit Care Med. 2016;194:317-26.

12. Kilic A, George TJ, Beaty CA, Merlo CA, Conte JV, Shah AS. The effect of center volume on the incidence of postoperative complications and their impact on survival after lung transplantation. J Thorac Cardiovasc Surg. 2012;144: $1502-8$.

13. Martin AN, Hassinger TE, Kozower BD, Camacho F, Anderson RT, Yao N, Disparities in lung cancer screening availability: lessons from Southwest Virginia. Ann Thorac Surg. 2019;108:412-6.

14. Suzuki Y, Cantu E, Christie JD. Primary graft dysfunction. Semin Respir Crit Care Med. 2013;34:305-9.

15. Mooney JJ, Bhattacharya J, Dhillon GS. Effect of broader geographic sharing of donor lungs on lung transplant waitlist outcomes. J Heart Lung Transplant. 2019; 38:136-44.

16. Canadian Society of Transplantation. Transplant programs \& OPOs. Available at: https://www.cst-transplant.ca/cgi/page.cgi/transplant-programs-opos.html. Accessed September 14, 2020.

17. Breathe: The Lung Association (Canadian Lung Association). Lung transplant information. Available at: https://ns.lung.ca/our-programs/lung-transplantinformation. Accessed September 14, 2020.

Key Words: lung transplantation, lung allocation, lung donation 